\title{
3 Forschungsfragen und Ziele der Arbeit
}

Im vorangegangenen Kapitel wurde das funktionale Denken als eine für den Umgang mit Funktionen typische Denkweise (Vollrath, 1989) vorgestellt und dabei auf verschiedene Möglichkeiten der dabei zentralen Denk- und Handlungsweisen eingegangen. Darüber hinaus wurden in Abschnitt 2.2 unterschiedliche Förderansätze beschrieben, deren Fokus hauptsächlich auf innermathematischen Betrachtungen lag. Ausgehend von einem fachübergreifenden Ansatz ist es ein Ziel der Arbeit, die Möglichkeit einer Förderung im biologischen Fachunterricht zu betrachten. Daraus ergibt sich die erste Forschungsfrage dieser Arbeit:

(F1) Inwiefern lässt sich funktionales Denken im biologischen Fachunterricht fördern?

Die Beantwortung dieser Frage soll exemplarisch an einem Themengebiet der Sekundarstufe II erfolgen.

Ein entsprechender Ansatz beinhaltet, mathematische Betrachtungen in den biologischen Fachunterricht zu integrieren. Wie in Abschnitt 2.3 beschrieben, wird jedoch für Mathematisierungen im Biologieunterricht gefordert, dass sie sich lediglich auf solche Bereiche beschränken sollen, in denen durch sie das Verständnis erleichtert wird (Horn, 2008). Daher ist ein weiteres Ziel dieser Arbeit, die Auswirkungen einer Förderung des funktionalen Denkens auf den biologischen Wissenserwerb zu untersuchen. Dementsprechend ergibt sich die zweite Fragestellung dieser Arbeit als:

(F2) Inwiefern beeinflusst ein Förderansatz zum funktionalen Denken im biologischen Fachunterricht den Erwerb von biologischem Fachwissen?

Hierfür wird ein entsprechender Test konzipiert, der es ermöglichen soll, die Entwicklung einzelner Komponenten des funktionalen Denkens sowie des biologischen Fachwissens zu messen. 